\title{
Capacity and Cutoff Rate of Noncoherent FSK with Nonselective Rician Fading
}

\author{
WAYNE E. STARK, MEMBER, IEEE
}

\begin{abstract}
The capacity and cutofi rate of frequency-shift keying (FSK) modulation and noncoherent reception when the signal is subject to Rician fading are calculated. Both hard and soft decisions with maximum likelihood combining are considered, as well as soft decisions with square-law combining. Optimal code rates are found that minimize the required signal-to-noise ratio for reliable communication.
\end{abstract}

\section{INTRODUCTION}

$\mathrm{T}$ HE use of error-correcting codes has become widespread in the last several years in digital communication systems. Error-correcting codes are extremely useful in communication systems in which there is strong pulsed interference [1]-[4]. The improvement in signal-to-noise ratio with coding can be on the order of $30 \mathrm{~dB}$ for these channels. Another communication channel for which there are large potential gains by the use of error-correction coding is the Rayleigh fading channel. This channel is very similar qualitatively to the pulsed interference channel [1]. For this channel, without the use of coding, the average bit error probability varies (approximately) inversely with the signal-to-noise ratio. This is in contrast to the case of white Gaussian noise, where the error probability is an exponentially decreasing function of the signal-to-noise ratio. It is shown in [5] that with simple repetition codes (i.e., diversity transmission) on a Rayleigh faded channel, the coded error probability can be made to decrease exponentially with the signal-to-noise ratio (when the diversity level or length of the repetition code is chosen optimally). It is also shown [5, p. 550] that for repetition coding, the loss incurred by a Rayleigh faded channel over a nonfaded channel is approximately $5.25 \mathrm{~dB}$. However, for very low error probability the rate of the optimal length repetition code becomes very small. The possibility of having very small error probability without the rate of the code approaching zero as the block length becomes very large is guaranteed when the channel capacity is nonzero. We are interested in the largest possible rate for which reliable communication (arbitrarily small error probability) is possible, or equivalently the smallest possible signal-to-noise ratio which guarantees arbitrarily small error probability with codes of rate $r$. Here we show that the loss due to fading can be reduced to approximately $1.35 \mathrm{~dB}$ by the use of optimal codes. Furthermore, these codes have nonzero rate and have arbitrarily small error probability. (The loss due to fading will be different when we do not require arbitrarily reliable communication, and could in fact be negative.)

In this paper we examine the performance of optimal codes on a channel subject to Rician fading as measured by the minimum signal-to-noise ratio necessary for reliable communications. This minimum can be calculated by computing the

Paper approved by the Editor for Communication Theory of the IEEE Communications Society. Manuscript received August 20, 1984; revised May 22, 1985. This paper was presented at the International Symposium on Information Theory, St. Jovite, P.Q., Canada, September 1983. This work was supported in part by the National Science Foundation under Grant ECS8307150 .

The author is with the Department of Electrical Engineering and Computer Science. University of Michigan, Ann Arbor, MI 48105. channel capacity. We also examine the "practical limits" [6] of coding by computing the cutoff rate. The cutoff rate is the rate above which the number of steps per decoded digit becomes infinite with sequential decoding [5]. The cutoff rate is also useful in determining the error probability of convolutional codes [7].

We first discuss some of the assumptions that will be made and their implications on the results. The modulation we consider is that of $M$-ary FSK; which generates one of $M$ orthogonal signals of duration $T$. The received signal consists of three components: the transmitted signal (with some constant attenuation $\alpha$ and a constant phase), the transmitted signal with the attenuation $R$ being Rayleigh distributed and phase uniformly distributed on the interval $[0,2 \pi]$, and a white Gaussian noise component that is independent of the other random quantities. Since we assume an unknown phase for the received signal, we are considering a noncoherent communication system. We consider soft decision (maximumlikelihood) receivers for the binary case and hard decision receivers for the $M$-ary case.

We will assume that the channel is memoryless and that the random attenuation $R$ is constant during every symbol duration. The memoryless assumption is equivalent to assuming that the random variables that determine the attenuation during a symbol transmission are independent from symbol to symbol. In reality, this attenuation is a slowly varying function of time which could be modeled as a slowly varying randoin process; however, by assuming that the channel is memoryless, we obtain lower bounds on the capacity of the channel. From the results in [8] we know that if the receiver has an additional output, the attenuation $R$ of the channel (which is Rayleigh distributed), then the capacity with memory is independent of the memory length. Also, the capacity without this "side information" is less than the capacity with side information, and approaches the capacity with side information as the memory length becomes large. Thus, by computing the capacity under the memoryless assumption with and without side information, we obtain bounds on the capacity with memory. The actual evaluation of the capacity of channels with memory and hard decision receivers has been carried out in [9]. Here we only present results for the memoryless case. Since the capacity with side information is larger than the capacity without, one conclusion that is reached in [9] is that interleaving decreases the channel capacity unless side information is available. The interested reader should consult [9] for further discussion of this.

The coding theorem of information theory guarantees there exist codes with rate less than the capacity, such that the error probability can be made arbitrarily small when decoded using maximum likelihood decoding. For a Rician faded channel, maximum likelihood decoding is very hard to implement since it involves highly nonlinear functions. A receiver that can be more easily implemented is the square-law combining receiver. For this receiver we are interested in knowing what is the largest rate for which reliable communication is possible. This is given by the "mismatched" capacity which is defined in [10] and [11]. We examine the mismatch capacity for Rician fading when using square-law combining. Although square- 
law combining is suboptimal, we show that the theoretical loss in using square-law combining is very small (less than $0.1 \mathrm{~dB}$ ).

The outline of the paper is as follows. In Section II we describe mathematically the model of a Rician faded channel. In Section III we calculate the capacity of a Rician fading channel with both soft and hard decisions. We then calculate the capacity of a Rayleigh faded channel with side information available. Last; we calculate the "mismatched" capacity when using square-law combining. In Section IV we repeat these calculations for the cutoff rate. We see that the cutoff rate with square-law combining is closely related to the Chernoff bound on error probability. Numerical results are discussed in Section $\mathrm{V}$, where we give the code rates that minimize the
For the Rician fading channel model considered, it is easy to show that the probability densities of the random variables $Y_{k, c}$ and $Y_{k, s}$ conditioned on $X=i$ are Gaussian with mean

$$
\begin{aligned}
& E\left[Y_{k, c} \mid X=i\right]=\alpha \beta \cos \theta_{k} \delta_{i, k} \\
& E\left[Y_{k, s} \mid X=i\right]=\alpha \beta \sin \theta_{k} \delta_{i, k}
\end{aligned}
$$

where $\delta_{i, k}=1$ if $i=k$ and is zero otherwise and $\beta^{2}=2 E_{T} / N_{0}$. The variances of $Y_{k, c}$ and $Y_{k, s}$ are

$$
\operatorname{var}\left[Y_{k, c} \mid X=i\right]=\operatorname{var}\left[Y_{k, s} \mid X=i\right]=\dot{\sigma}^{2} \beta^{2} \delta_{i, k}+1 \text {. }
$$

The conditional density function $Y_{k}$ is then given by

$$
p\left(y_{k} \mid X=i\right)= \begin{cases}\frac{1 / 2}{1+\sigma^{2} \beta^{2}} \exp \left\{-\frac{1}{2} \frac{y_{k}+\alpha^{2} \beta^{2}}{1+\sigma^{2} \beta^{2}}\right\} I_{0}\left\{\frac{\sqrt{\alpha^{2} \beta^{2} y_{k}}}{1+\sigma^{2} \beta^{2}}\right\}, & k=i \\ 1 / 2 \exp \left\{-\frac{1}{2} y_{k}\right\}, & k \neq i\end{cases}
$$

signal-to-noise ratio necessary for reliable communication.

\section{Channel Models}

In this section we present the channel models for frequencyshift keying used in this paper. The input alphabet $A$ for the channel consists of $M$ letters (say $A=\{0,1, \cdots, M-1\}$ ). If $X$ is the input to the channel, then $X=i$ corresponds to transmitting a sine wave $s_{i}(t)$ with power $P$ for $T$ seconds at frequency $\omega_{i}$ :

$$
s_{i}(t)=\sqrt{2 P} \cos \left(\omega_{i} t+\theta_{i}\right), \quad 0 \leq t \leq T
$$

where $\dot{\theta}_{i}$ is the phase of the $i$ th signal. (We assume throughout that $\omega_{i}, 0 \leq i \leq M-1$, and $T$ are chosen so that the $s_{i}(t)$ form an orthogonal signal set.)

For the fading channel, when the transmitted signal is $s_{i}(t)$, the received signal is given by

$$
\dot{r}(t)=\alpha s_{i}(t)+R \sqrt{2 P} \cos \left(\omega_{i} t+\theta_{i}+\phi\right)+n(t)
$$

where $\alpha$ is the strength of the specular component, and $R$ is a Rayleigh distributed random variable with mean square $2 \sigma^{2}$ :

$$
p_{R}(r)=\left\{\begin{array}{cc}
\frac{r}{\sigma^{2}} e^{-r^{2} / 2 \sigma^{2}} & r \geq 0 \\
0 & r<0 .
\end{array}\right.
$$

Also, in (2), $\phi$ is uniformly distributed between 0 and $2 \pi$ and independent of $R$. The noise $n(t)$ in (2) is a white Gaussian noise process with two-sided spectral density $N_{0} / 2$. Setting $\alpha$ $=0$ in (2) results in the slow Rayleigh fading channel. Setting $2 \sigma^{2}=0$ yields the nonfaded model. Letting $\gamma^{2}=2 \sigma^{2} / \alpha^{2}$, we have the Rayleigh model for $\gamma^{2}=\infty$ and the nonfading case for $\gamma^{2}=0$. For $0<\gamma^{2}<\infty$ we have the Rician fading channel. The average received energy per transmission is given by $\bar{E}_{T}=\left(\alpha^{2}+2 \sigma^{2}\right) E_{T}$ where $E_{T}=P T$.

The receiver computes the $M$-dimensional vector $Y=\left(Y_{0}\right.$, $\left.\cdots, Y_{M-1}\right)$ where

$$
\begin{gathered}
Y_{i}=Y_{i, c}^{2}+Y_{i, s}^{2} \\
Y_{i, c}=\frac{2}{\sqrt{N_{0} T}} \int_{0}^{T} r(t) \cos \omega_{i} t d t \\
Y_{i, s}=\frac{2}{\sqrt{N_{0} T}} \int_{0}^{T} r(t) \sin \omega_{i} t d t .
\end{gathered}
$$

for $y \geq 0$ and $p\left(y_{k} \mid X=i\right)=0$ for $y_{k}<0$. In (5) $I_{0}$ is the modified Bessel function of order zero. Note that if $\Gamma=\bar{E}_{T} /$ $N_{0}$ and $\gamma^{2}=2 \sigma^{2} / \alpha^{2}$, then we have $\alpha^{2} \beta^{2}=2 \Gamma /\left(1+\gamma^{2}\right)$ and $\sigma^{2} \beta^{2}=\gamma^{2} \Gamma /\left(1+\gamma^{2}\right)$. Using the above relations in (5) we can express $p\left(y_{k} \mid X=i\right)$ in terms of $\Gamma$ and $\gamma^{2}$.

\section{Capacity of Memoryless Fading Channels}

In this section we calculate the capacity of FSK with noncoherent reception in the presence of Rician fading. We consider several possible situations. First, we calculate the capacity of the Rician fading channel when the receiver uses maximum likelihood (optimum) decoding. We calculate this for both hard and soft decision receivers. Next, we consider the case that the receiver uses a square-law combining receiver. We calculate the loss incurred by such a suboptimal receiver. Last, we consider a Rayleigh faded channel and calculate the capacity when the receiver has side information concerning the level of fade for each received symbol. This is done for both hard and soft decision receivers.

The capacity of a memoryless channel with input $X$ and output $Y$ is given by

$$
\begin{aligned}
C & =\max _{X} I(X ; Y) \\
& =\max _{X} \sum_{i=0}^{M-1} \int_{y} p(y \mid X=i) P\{X=i\} \log _{2} \frac{p(y \mid X=i)}{p(y)} d y
\end{aligned}
$$

where the maximum is over all distributions on the random variable $X$ and the integration is over $M$. Due to the symmetry of the channel we consider, the distribution that achieves the maximum in (6) is the uniform distribution with $P\{X=i\}=$ $1 / M$ for $i=0,1, \cdots, M-1$, so that we can write the capacity as

$$
C=1-\int_{y} p(y \mid X=0) \log _{M}\left[1+\sum_{j=1}^{M-1} \Lambda_{j}(y)\right] d y
$$

where

$$
\Lambda_{j}(y)=\frac{p(y \mid X=j)}{p(y \mid X=0)} .
$$

For the Rician faded channel, $p(y \mid X=i)$ can be determined 
from (5) as

$$
p(y \mid X=i)=\frac{f\left(y_{i}\right) 2^{-M}}{\left(1+\sigma^{2} \beta^{2}\right)} \exp \left\{-\frac{1}{2}(\bar{y})-\frac{1}{2} \frac{\alpha^{2} \beta^{2}}{1+\sigma^{2} \beta^{2}}\right\}
$$

where $\bar{y}=\sum_{i=0}^{M-1} y_{i}$ and

$$
f(y)=\exp \left\{\frac{y \sigma^{2} \beta^{2}}{2\left(1+\sigma^{2} \beta^{2}\right)}\right\} I_{0}\left[\frac{\sqrt{\alpha^{2} \beta^{2} y}}{1+\sigma^{2} \beta^{2}}\right]
$$

so that

$$
\Lambda_{j}(y)=\frac{f\left(y_{j}\right)}{f\left(y_{0}\right)} .
$$

The capacity is thus

$$
\begin{array}{r}
C(\Gamma, \gamma)=1-\frac{\exp \left\{\frac{-\alpha^{2} \beta^{2} / 2}{1+\sigma^{2} \beta^{2}}\right\}}{2^{M}\left(1+\sigma^{2} \beta^{2}\right)} \int_{0}^{\infty} \cdots \int_{0}^{\infty} e^{-\hat{y} / 2} f\left(y_{0}\right) \\
\cdot \log _{M}\left[1+\sum_{j=1}^{M-1} \Lambda_{j}(y)\right] d y .
\end{array}
$$

These integrals can be evaluated using Gaussian-Laguerre quadratic formulas [4], but the evaluation requires large amounts of computation when $M>2$. For the special case of no fading (i.e., additive white Gaussian noise channel), the capacity is found by letting $\gamma^{2}=0.0$ :

$$
\begin{aligned}
C(\Gamma, 0)=1-\frac{e^{-\alpha^{2} \beta^{2} / 2}}{2^{M}} \int_{0}^{\infty} & \cdots \int_{0}^{\infty} e^{-y / 2} I_{0}\left[\sqrt{\alpha^{2} \beta^{2} y_{0}}\right] \\
\cdot \log _{M} & {\left[1+\sum_{j=1}^{M-1} \Lambda_{j}(y)\right] d y }
\end{aligned}
$$

and

$$
\Lambda_{j}(y)=\frac{I_{0}\left[\sqrt{\alpha^{2} \beta^{2} y_{j}}\right]}{I_{0}\left[\sqrt{\alpha^{2} \beta^{2} y_{0}}\right]}
$$

which for $M=2$ is a result in [12]. For the special case of Rayleigh fading, the capacity is found by letting $\gamma^{2}=\infty$ or $\alpha$ $=0$ :

$$
\begin{aligned}
C(\Gamma, \infty)=1 & -\frac{1}{2^{M}\left(1+\sigma^{2} \beta^{2}\right)} \int_{0}^{\infty} \cdots \int_{0}^{\infty} e^{-\frac{\bar{y}}{2}+\frac{y_{0} \sigma^{2} \beta^{2}}{2\left(1+\sigma^{2} \beta^{2}\right)}} \\
& \cdot \log _{M}\left\{1+\exp \left[\frac{\left(\bar{y}-2 y_{0}\right) \sigma^{2} \beta^{2}}{2\left(1+\sigma^{2} \beta^{2}\right)}\right]\right\} d y .
\end{aligned}
$$

For hard decisions, the capacity of $M$-ary FSK with Rician fading can be easily computed. From [13] we have that the probability of error $P_{e}$ for Rician fading is given by

$$
P_{e}=\sum_{j=1}^{M-1} \frac{(-1)^{j+1}}{j+1+j \sigma^{2} \beta^{2}}\left(\begin{array}{c}
M-1 \\
j
\end{array}\right) \exp \left\{-\frac{j \alpha^{2} \beta^{2}}{j+1+j \sigma^{2} \beta^{2}}\right\}
$$

Since the signal set is an orthogonal set, the channel is an $M$ ary symmetric channel with crossover probability $P_{e} /(M-$ 1). The capacity $C_{M}(\Gamma, \gamma)$ for this channel in $M$-ary symbols/ channel use is achieved by uniform input distribution, and is given by $\tilde{C}_{M}(\Gamma, \gamma)=\hat{C}_{M}\left(P_{e}\right)$ where

$$
\hat{C}_{M}(x)=1+(1-x) \log _{M}(1-x)+x \log _{M} \frac{x}{M-1} .
$$

For a Rayleigh faded channel $[\alpha=0$ in (1)], we can compute the capacity when the receiver/decoder has a perfect estimate of the fading variable $R$ for each received symbol. In order to compute the capacity with this side information available to the decoder, we need to determine the joint density of the random vector $Y=\left(Y_{0}, Y_{1}\right)$ and the random variable $R$. We first compute the conditional density of $Y_{k}$ given $R$. This can be seen to be

$p\left(y_{k} \mid R, X=i\right)$

$$
= \begin{cases}\frac{1}{2} e^{-\left(y_{k}+R^{2} \beta^{2}\right) / 2} I_{0}\left(\sqrt{y_{k} R^{2} \beta^{2}}\right) & k=i \\ \frac{1}{2} e^{-y_{k} / 2} & k \neq i\end{cases}
$$

for $y_{k} \geq 0$ and $p\left(y_{k} \mid R, X=i\right)=0$ for $y_{k}<0$. The joint density of $Y$ given $R$ and $X=i$ is then

$p(y \mid R, X=i)=2^{-M}$

$$
\cdot \exp \left\{-\frac{1}{2}\left(\bar{y}+R^{2} \beta^{2}\right)\right\} I_{0}\left(\sqrt{y_{i} R^{2} \beta^{2}}\right)
$$

for $y_{i} \geq 0, i=0,1, \cdots, M-1$, and is 0 otherwise. The joint density of $Y, R$ can be found from (3) and (14) as

$p(y, r \mid X=i)=\frac{r}{2^{M} \sigma^{2}}$

$$
\cdot \exp \left\{-\frac{1}{2}\left(\bar{y}+r^{2}\left(\beta^{2}+\frac{1}{\sigma^{2}}\right)\right)\right\} I_{0}\left(\sqrt{y_{i} r^{2} \beta^{2}}\right) .
$$

The capacity with side information available is then

$$
\begin{aligned}
\bar{C}=1-\int_{y_{0}=0}^{\infty} \cdots \int_{y_{M-1}=0}^{\infty} \int_{r=0}^{\infty} p(y, r \mid X=0) \\
\cdot \log _{2}\left(1+\sum_{j=1}^{M-1} \Lambda_{j}(y, r)\right) d y d r
\end{aligned}
$$

where

$$
\Lambda_{j}(y, r)=\frac{p(y, r \mid X=j)}{p(y, r \mid X=0)} .
$$

With a hard decision receiver, the capacity with side information available can be computed using results in [8] as

$$
C=\int_{r=0}^{\infty} \frac{r}{\sigma^{2}} e^{-r^{2} / 2 \sigma^{2}}\left[C_{M}\left(P_{e}(r)\right)\right] d r
$$

where

$$
P_{e}(r)=\sum_{j=1}^{M-1} \frac{(-1)^{j+1}}{j+1}\left(\begin{array}{c}
M-1 \\
j
\end{array}\right) \exp \left\{-\frac{j r^{2} \beta^{2}}{j+1}\right\}
$$


is the conditional error probability given $R=r$, and $C_{M}(x)$ is given in (12). Numerical results will be given for these in Section V.

The capacity was determined for the Rician faded channel, based on assuming an optimal receiver. For Rician fading in general, this involves using a metric that involves the Bessel function and exponential functions which are not easily implemented in practice. A more practical receiver might instead just use the outputs $Y_{i}$ defined in (4) as the sum of the squares of the matched filter outputs. This receiver (decoder), which is called the square-law combining receiver, is suboptimal except when the fading is Rayleigh $(\alpha=0)$. Thus, a question that can be asked is what is the largest rate, such that codes exist with arbitrarily small error probability when using square-law combining (a suboptimal receiver). The question is a special case of a more general question about the largest rate for which reliable communication is possible when using a decoder with a decision rule based on the likelihood ratio between probabilities that are different from the actual channel transition probabilities. This has been answered by Fisher [11] and, for a symmetric channel, is given by

$$
C^{*}=1-\int_{y} p(y \mid X=0) \log _{M}\left(1+\sum_{j=1}^{M-1} \Lambda_{j}^{*}(y)\right) d y
$$

where

$$
\Lambda_{j}^{*}(y)=\frac{p^{*}(y \mid X=j)}{p^{*}(y \mid X=0)}
$$

and $p^{*}$ denotes the transition probabilities used by the decoder. When the receiver uses square-law combining, we let

$$
p^{*}(y \mid X=j)=\frac{1}{2^{M} a b^{M-1}} \exp \left\{-\frac{1}{2}\left(\frac{\bar{y}}{b}+y_{j} \frac{(b-a)}{a b}\right)\right\}
$$

for $y_{i} \geq 0, j=0,1, \cdots, M-1$ and $p^{*}(y \mid X=j)^{*}=0$ otherwise. Also in (20), $a$ and $b$ are positive parameters. Thus,

$$
\Lambda_{j}^{*}(y)=\exp \left\{-\frac{1}{2}\left(y_{j}-y_{0}\right)\left(\frac{b-a}{a b}\right)\right\}
$$

Notice when $a, b \in R^{+},(a-b) / a b$ can take on any value in $\boldsymbol{R}$. We let $z=(a-b) / a b$. The capacity under mismatch with parameter $z$ is then

$$
\begin{gathered}
C^{*}(z)=1-\int_{y} p(y \mid X=0) \log _{2} \\
\cdot\left(1+\sum_{j=1}^{M-1}, \exp \left\{\frac{1}{2}\left(y_{j}-y_{0}\right) z\right\}\right) d y .
\end{gathered}
$$

Since $z$ is a free parameter, we may choose $z$ to maximize $C^{*}(z)$. It is easy to show that $C^{*}(z)$ is a concave function of $z$ with $\lim _{z \rightarrow \infty} C^{*}(z)=\lim _{z \rightarrow-\infty} C^{*}(z)=-\infty, C^{*}(0)=0$, and $\partial C^{*}(z) /\left.\partial z\right|_{z=0} \geq 0$. From the above facts we conclude that $C^{*}(z)$ has a unique maximum that occurs for $z>0$. This maximum can be easily found numerically. We indicate the numerical results in the last section.

\section{Cutoff Rate of Memoryless Fading Channels}

In this section we evaluate the cutoff rate for the Rician fading channel with both soft and hard decisions. We compare this to the cutoff rate when using a square-law combining receiver which is suboptimal if there is a nonzero direct path component in the received signal. For completeness, we also evaluate the cutoff rate in the Rayleigh fading case when the receiver has knowledge about the strength of the received signal which has been calculated earlier by Bucher [14] for $M$ $=8$. Here we give formulas for calculating the cutoff rate for general $M$. This is done for hard and soft decision receivers.

The cutoff rate when using $M$ orthogonal signals and maximum likelihood decoding has been shown to be given by [15]

$$
R_{0}=1-\log _{M}[1+(M-1) D]
$$

where

$$
D=\left[\int_{-\infty}^{\infty} \sqrt{p\left(y_{0} \mid X=0\right) p\left(y_{0} \mid X=1\right)} d y_{0}\right]^{2} .
$$

To compute the cutoff rate for Rician fading, we need only substitute (5) into (23). Thus,

$$
\begin{aligned}
R_{0}=1 & -\log _{M}\left[1+(\mathrm{M}-1)\left\{\int_{0}^{\infty} \frac{1}{2 \sqrt{1+\sigma^{2} \beta^{2}}} \exp \right.\right. \\
& \cdot\left(-\frac{1}{4}\left[\frac{2+\sigma^{2} \beta^{2}}{1+\sigma^{2} \beta^{2}}\right] y_{0}-\frac{1}{4} \frac{\alpha^{2} \beta^{2}}{1+\sigma^{2} \beta^{2}}\right) \\
& \left.\left.\cdot \sqrt{I_{0}\left(\sqrt{\alpha^{2} \beta^{2} y_{0}} /\left(1+\sigma^{2} \beta^{2}\right)\right) d y_{0}}\right\}^{2}\right] .
\end{aligned}
$$

This is the cutoff rate for noncoherent FSK with maximum likelihood decoding and soft decisions. For the special case of Rayleigh fading, (24) reduces to

$$
R_{0}=1-\log _{M}[1+(M-1) 4 p(1-p)]
$$

where $p=\left(2+\sigma^{2} \beta^{2}\right)^{-1}$. If, in addition to the vector $Y$ as the channel output, the receiver also has knowledge of the random variable $R$ for each received symbol, then the receiver has side information. In this case the cutoff rate for Rayleigh fading can be shown to be

$$
\bar{R}_{0}=1-\log _{M}[1+(M-1) \bar{D}]
$$

where

$$
\bar{D}=\int_{0}^{\infty} \frac{r}{\sigma^{2}} e^{-r^{2} / 2 \sigma^{2}} D(r) d r
$$

and

$$
\begin{aligned}
D(r)= & {\left[\int_{0}^{\infty} \frac{1}{2}\right.} \\
& \left.\cdot \exp \left\{-\frac{1}{2}\left(y_{0}+r^{2} \beta^{2} / 2\right)\right\} \sqrt{I_{0}\left(\sqrt{y_{0} r^{2} \beta^{2}}\right)} d y_{0}\right]^{2} .
\end{aligned}
$$

Notice that for $M$ orthogonal signaling with or without side information, $D$ does not depend on $M$, the number of orthogonal signals. As a result, once $D$ is calculated (for each signal-to-noise ratio), we can calculate the cutoff rate for every $M$. This is not true when hard decisions are made, as shown below.

When hard decisions are made, the cutoff rate is

$$
R_{0}=1-\log _{M}\left(1+(M-1) \hat{D}\left(P_{e}\right)\right)
$$

where

$$
\hat{D}(x)=\frac{M-2}{M-1} x+2 \sqrt{x(1-x) /(M-1)}
$$


and $P_{e}$ is given in (11). When side information is available, the cutoff rate with hard decisions (on $y$ ) becomes [8]

$$
\bar{R}_{0}=1-\log _{M}(1+(M-1) \bar{D})
$$

where

$$
\bar{D}=\int_{0}^{\infty} \frac{r}{\sigma^{2}} e^{-r^{2} / 2 \sigma^{2}} \hat{D}\left(P_{e}(r)\right) d r
$$

with $P_{e}(r)$ given in (18).

Finally, we evaluate the cutoff rate with Rician fading under mismatch (i.e., square-law combining). The cutoff rate under mismatch was defined in [16] as

$$
R_{0}^{*}=1-\log _{M}\left(1+(M-1) D^{*}\right)
$$

where

$$
D^{*}=\min _{\gamma \geq 0} \int_{y} p(y \mid X=0)\left[\frac{p^{*}(y \mid X=1)}{p^{*}(y \mid X=0)}\right]^{\lambda} d y .
$$

Using (21) in (31), we obtain

$$
\begin{aligned}
D^{*} & =\min _{\lambda \geq 0} \int_{y} p(y \mid X=0) \exp \left\{-\frac{1}{2} \hat{\lambda}\left(y_{0}-y_{1}\right)\right\} d y \\
& =\min _{\lambda \geq 0} E\left[e^{-1 / 2 \lambda\left(y_{0}-y_{1}\right)} \mid X=0\right] .
\end{aligned}
$$

We now see that (32) is the Chernoff bound on error probability for a Rician faded channel. This has been computed by Jacobs [17] as

$$
D^{*}=\frac{\exp \left\{a w\left[1+w\left(1+\sigma^{2} \beta^{2}\right)\right]\right\}}{1+w \sigma^{2} \beta^{2}-w^{2}\left(1+\sigma^{2} \beta^{2}\right)}
$$

where

$$
w=\frac{\sqrt{a^{2}+6 a x y+x^{2} y^{2}}-a-y z}{4 y^{2}}
$$

and $a=\alpha^{2} \beta^{2} / 2, x=2+\sigma^{2} \beta^{2}, y=1+\sigma^{2} \beta^{2}$, and $z=2-$ $\sigma^{2} b$. Numerical results for the cutoff rate will be given in the next section. For the case of Rayleigh fading where square-law combining is optimum, (33) and (30) reduce to (25).

\section{Numerical Results and Discussion}

In this section we present some numerical results for the cutoff rate and capacity. We first relate the symbol signal-tonoise ratio $E / N_{0}$ to the information bit signal-to-noise ratio $E_{b} / N_{0}$. If we use codes of rate $r$ information symbols/channel symbol, then we have that

$$
\frac{E_{b}}{N_{0}}=\frac{E}{N_{0}} /\left(r \log _{2} M\right) .
$$

From the channel coding theorem [18], there exist codes of rate $r$ with arbitrarily small error probability, provided $r$ is less than the channel capacity. The capacity is a function of the bit signal-to-noise ratio, so reliable communications is possible, provided

$$
r \leq C\left(E / N_{0}\right)
$$

or

$$
\frac{E_{b}}{N_{0}} \geq \frac{C^{-1}(r)}{r \log _{2} M} .
$$

The right-hand side of $(34)$ is the minimum signal-to-noise ratio (over all possible codes of rate $r$ ) necessary for reliable

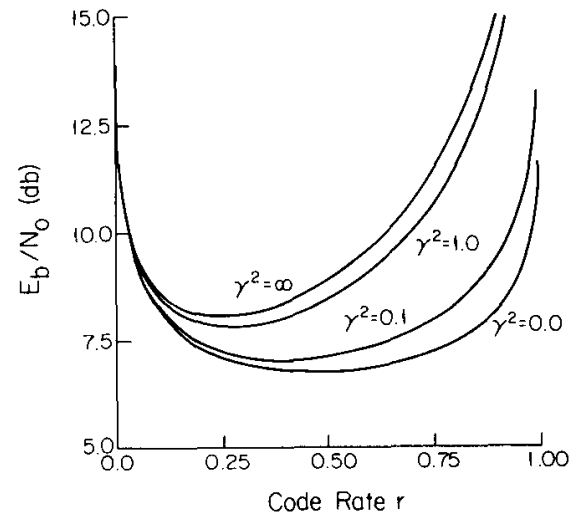

Fig. 1. $E_{b} / N_{0}$ needed for reliable communication for Rician fading with soft decisions.

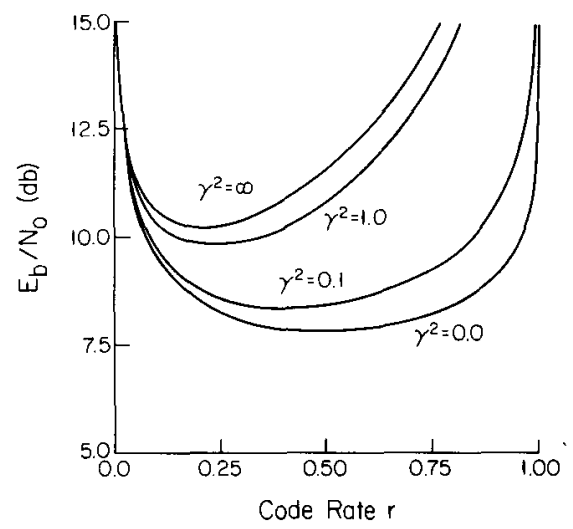

Fig. 2. $E_{b} / N_{0}$ needed for reliable communication for Rician fading with hard decisions.

communications. Similar calculations for the cutoff rate show "practical communications" are possible with codes of rate $r$, provided

$$
\frac{E_{b}}{N_{0}} \geq \frac{R_{0}^{-1}(r)}{r \log _{2} M} .
$$

In Figs. 1-6 we plot the right-hand side of (34) and (35) as a function of the code rate $r$ for various cases. In Fig. 1 the minimum signal-to-noise ratio for reliable communications with Rician fading and a soft decision receiver is shown. The corresponding results for a hard decision receiver are shown in Fig. 2. In Fig. 3 we show the minimum signal-to-noise ratio for reliable communications with Rayleigh fading $\left(\gamma^{2}=\infty\right)$ for four receivers. These are soft and hard decision receivers with and without side information available to the decoder. Evidently, side information is worth considerably more in the hard decision receiver than in the soft decision receiver. However, the decoder in the case of side information available and hard decisions (on $y$ ) does not quantize the side information. These curves with and without side information also give an indication of the signal-to-noise ratio needed for reliable communication with codes of rate $r$ when the channel has memory. The signal-to-noise ratio necessary for reliable communications when the channel has memory is upper bounded by the signal-to-noise ratio needed in the memoryless case, and is lower bounded by the signal-to-noise ratio needed when side information is available.

We notice from these curves that there exists an optimal code rate for which the signal-to-noise ratio necessary for reliable communication is minimized. Notice also that the curves are relatively flat near the optima for the AWGN 


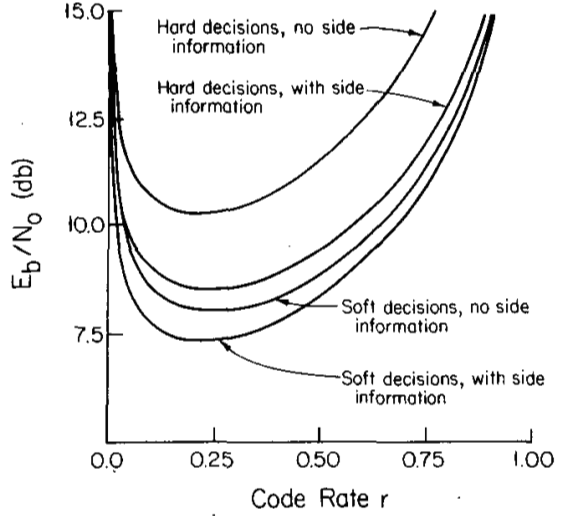

Fig. 3. $E_{b} / N_{0}$ needed for reliable communication for Raleigh fading with and without side information available (hard and soft decisions).

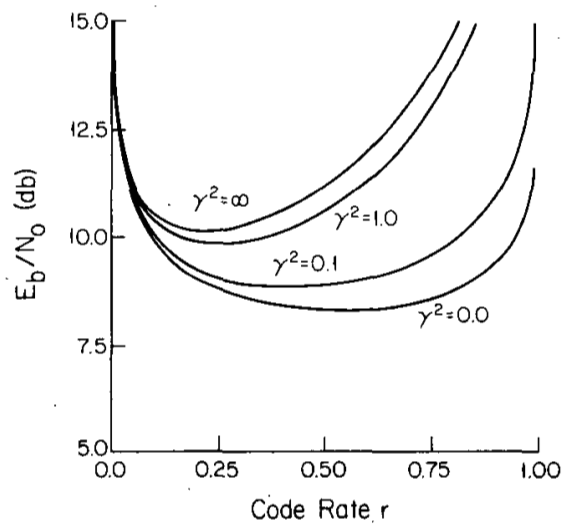

Fig. 4. $E_{b} / N_{0}$ needed for $R_{0}>r$ for Rician fading with soft decisions.

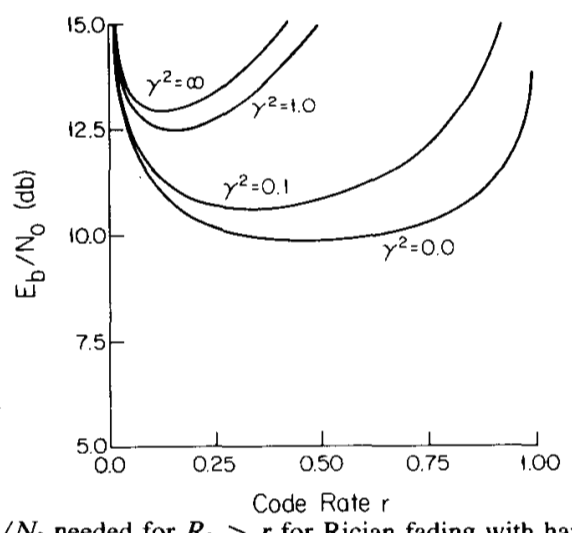

Fig. 5. $E_{b} / N_{0}$ needed for $R_{0}>r$ for Rician fading with hard decisions.

channel, compared to the Rayleigh fading channel. This indicates that the performance is much more sensitive to the code rate chosen in the Rayleigh fading channel, as compared to the AWGN channel. The optimal code rates are summarized in Table I. We also include the minimum signal-to-noise ratio for the case of square-law combining receiver. (We have not plotted the performance of the square-law combining receiver, since it is very close to the maximum-likelihood combining receiver.) From Table I we see that square-law combining is less efficient than maximum likelihood combining by less than $0.1 \mathrm{~dB}$. We also notice from Table I that the loss incurred by Rayleigh fading $\left(\gamma^{2}=\infty\right)$, compared to no fading $\left(\gamma^{2}=0.0\right)$, is $1.35 \mathrm{~dB}$. This is for the best codes (and for the optimal rate). This should be compared to the $5.25 \mathrm{~dB}$ loss [5] when comparing uncoded FSK with Gaussian noise and repetition coded FSK with Rayleigh fading.

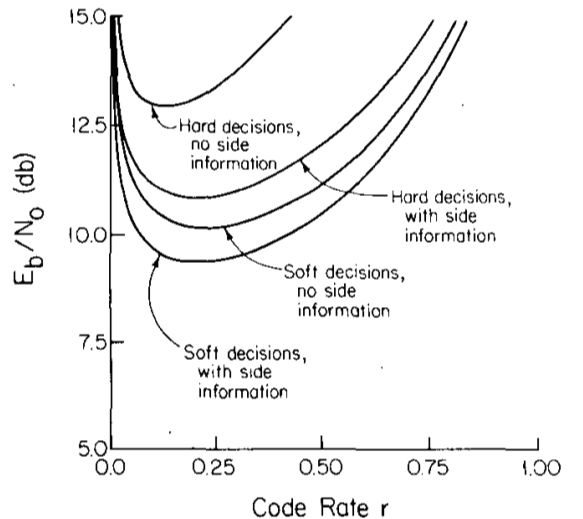

Fig. 6. $E_{b} / N_{0}$ needed to achieve cutoff for $R_{0}>r$ for Rayleigh fading with and without side information available (hard and soft decisions).

TABLE I

MINIMUM $E_{b} / N_{0}$ AND OPTIMAL CODE RATES FOR RELIABLE COMMUNICATION

\begin{tabular}{lccc}
\hline \hline & $\gamma^{2}$ & $E_{b} / N_{0}(\mathrm{~dB})$ & Rate \\
\hline Square-law combining & 0.0 & 6.78 & 0.47 \\
Maximum likelihood combining & 0.0 & 6.71 & 0.48 \\
Hard decisions & 0.0 & 7.82 & 0.50 \\
Maximum likelihood combining & $\infty$ & 8.06 & 0.24 \\
Maximum likelihood combining, & & & \\
$\quad$ side information available & $\infty$ & 7.37 & 0.23 \\
Hard decisions & $\infty$ & 10.24 & 0.21 \\
$\quad$ Hard decision, side & $\infty$ & 8.53 & 0.24 \\
$\quad$ information available & & & \\
\hline
\end{tabular}

TABLE II

MINIMUM $E_{b} / N_{0}$ AND OPTIMAL CODE RATES BASED ON THE CUTOFF RATE

\begin{tabular}{|c|c|c|c|c|}
\hline & * & $\gamma^{2}$ & $E_{b} / N_{0}(\mathrm{~dB})$ & Rate \\
\hline Square-law combining & & 0.0 & 8.44 & 0.52 \\
\hline Maximum likelihood combining & & 0.0 & 8.29 & 0.53 \\
\hline Hard decisions & & 0.0 & 9.84 & 0.48 \\
\hline Maximum likelihood combining & & $\infty$ & 10.14 & 0.23 \\
\hline \multicolumn{5}{|l|}{ Maximum likelihood combining, } \\
\hline Hard decisions & & $\infty$ & 12.94 & 0.13 \\
\hline \multicolumn{5}{|l|}{ Hard decision, side } \\
\hline information available & & $\infty$ & 10.84 & 0.20 \\
\hline
\end{tabular}

In Figs. 4-6 we show the corresponding results (to Figs. 13) when the cutoff rate is the parameter of interest instead of the channel capacity. The corresponding curves for other values of $M$ for soft decisions can be obtained using (23a) as follows. Let $f_{2}(r)$ be the minimum signal-to-noise ratio such that $R_{0}=r$ using two orthogonal signals. Then the minimum signal-to-noise ratio $f_{M}(r)$ for $M$ orthogonal signals is given by

$$
\begin{aligned}
f_{M}(r)=\frac{1}{r \log _{2} M} f_{2}\left(1-\log _{2}\right. & \left.\left(\frac{M^{1-r}-1}{M-1}+1\right)\right) \\
\cdot & {\left[1-\log _{2}\left(\frac{M^{1-r}-1}{M-1}+1\right)\right] . }
\end{aligned}
$$

In Table II, the optimal code rates are given with the minimum necessary signal-to-noise ratio. 


\section{REFERENCES}

[1] A. J. Viterbi and I. M. Jacobs, "Advances in coding and modulation for noncoherent channels affected by fading, partial band, and multipleaccess interference," in Advances in Communication Systems, vol. 4. New York: Academic, pp. 279-308, 1975.

[2] J. K. Omura and B. K. Levitt, " Coded error probability evaluation for antijam communication systems," IEEE Trans. Commun., vol. COM-30, pp. 896-903, May 1982.

[3] W. E. Stark, "Coding for frequency-hopped spread-spectrum communication with partial-band interference-Part II: Coded performance," IEEE Trans. Commun., vol. COM-33, pp. 1036-1044, Oct. 1985.

[4] M. B. Pursley and W. E. Stark, "Performance of Reed-Solomon coded frequency-hop spread-spectrum communications in partial-band interference," IEEE Trans. Commun., vol. COM-33, pp. 767-774, Aug. 1985 .

[5] J. M. Wozencraft and I. M. Jacobs, Principles of Communication Engineering. New York: Wiley, 1965.

[6] J. L. Massey, "Coding and modulation in digital communications," in Proc. Int. Zurich Sem. Digital Commun., Zurich, Switzerland, Mar. 1974 .

[7] A. J. Viterbi, "Error bounds for convolutional codes and an asymptotically optimum decoding algorithm," IEEE Trans. Inform. Theory, vol. IT-13, pp. 260-269, Apr. 1967.

[8] R. J. McEliece and W. E. Stark, "Channels with block interference," IEEE Trans. Inform. Theory, vol. IT-30, pp. 44-53, Jan. 1984

[9] W. E. Stark and R. J. McEliece, "On the capacity of channels with finite memory with applications to slowly fading channels," IEEE Trans. Inform. Theory, submitted for publication.

[10] M. Ohkubo, "Universal coding for multiple access channels," IEEE Trans. Inform. Theory, vol. IT-27, pp. 728-734, Nov. 1981.
[11] T. R. M. Fisher, "Some remarks on the role of inaccuracy in Shannon's theory of information transmission," in Trans. 8th Prag. Conf. Inform. Theory, 1978, pp. 211-226.

[12] S. A. Butman, I. Bar-David, B. K. Levitt, R. F. Lyon, and M. J. Klass, "Design criteria for noncoherent Gaussian channels with MFSK signaling and coding," IEEE Trans. Commun., vol. COM-24, pp. $1078-1088$, Oct. 1976.

[13] W. C. Lindsey, "Error probabilities for Rician fading multichannel reception of binary and $N$-ary signals," IEEE Trans. Inform. Theory, vol. IT-10, pp. 339-350, Oct. 1964.

[14] E. A. Bucher, "Coding options for efficient communication on nonstationary channels," in Nat. Telecommun. Conf. Rec., Dec. 1980, pp. 4.1.1-4.1.7.

[15] K. L. Jordan, "The performance of sequential decoding in conjunction with efficient modulation," IEEE Trans. Commun. Technol., vol, COM-14, pp. 283-297, June 1985.

[16] L. Biederman, J. K. Omura, and P. C. Jain, "Decoding with approximate channel statistics for band-limited nonlinear satellite channels," IEEE Trans. Inform. Theory, vol. IT-27, pp. 697-707, Nov. 1981.

[17] I. M. Jacobs, "Probability of error bounds for binary transmission on the slowly fading Rician channel," IEEE Trans. Inform. Theory, vol. IT-12, pp. 431-441, Oct. 1966

[18] C. E. Shannon, "A mathematical theory of communication," Bell Syst. Tech. J., vol. 27, pp. 623-656, Oct. 1948.

Wayne E. Stark (S'77-M'78), for a photograph and biography, see p. 774 of the August 1985 issue of this TRANSACTIONS. 